\title{
Multi Wavelength Interferometry for High Precision Distance Measurement
}

Petter, Jürgen

Luphos $\mathrm{GmbH}$

Landwehrstr. 55

64293 Darmstadt

Precision in mechanical fabrication increased drastically within the last years. In many areas of industrial fabrication these days the precision of production processes reaches beyond the scale of micrometers, while diamond grinded metallic surfaces can be manufactured with optical quality even without finishing polish. High precision optics, aspheric lenses or optical freeforms are produced with only nanometer tolerance while the positioning of bearings and object carriers (e.g. in wafer procession) needs subnanometer resolution. Such high accuracy demands an even higher precision in measurement technology being used e.g. for distance and topology measurements, for the positioning of axis or the quality control of fabricated objects.

Techniques that nowadays are available for these measurement problems are either complicated in adaption or implicate substantial disadvantages slowing down the fabrication process considerably. Futhermore, using tactile measurement systems in topological survey the sensor itself can harm the surface, especially when perfomred in the nanometer precision range; conventional optical measurement systems either are less precise or the dimesions of their sensorheads are to large to be handeled properly. In common they suffer from an only small and fixed workingrange, which makes them not applicable in different and changeable measurement requirements and distances and mutable working areas. Even though simple interferometric methods principly are able to measure long distances with high accuracy, it is done only relativlely by counting fringes. Moreover, due to the phase ambiguity they fail when measuring topologies of rough surfaces.

Compared with the aforementioned distance maesurement systems the newly developped Multiwavelength-Interferometer (MWLI) by Luphos offeres a flexible alternative for high precision measurements for distances and topologies. Using the special method of three interferometers folded into each other the disadvantages of common distance measurement systems are being omitted. The system with its small fibrecoupled sensorhead offers a large flexibility and enables a versatile implementation of the sensor in a whole new area of applications, where measurement hasn't been possible so far.

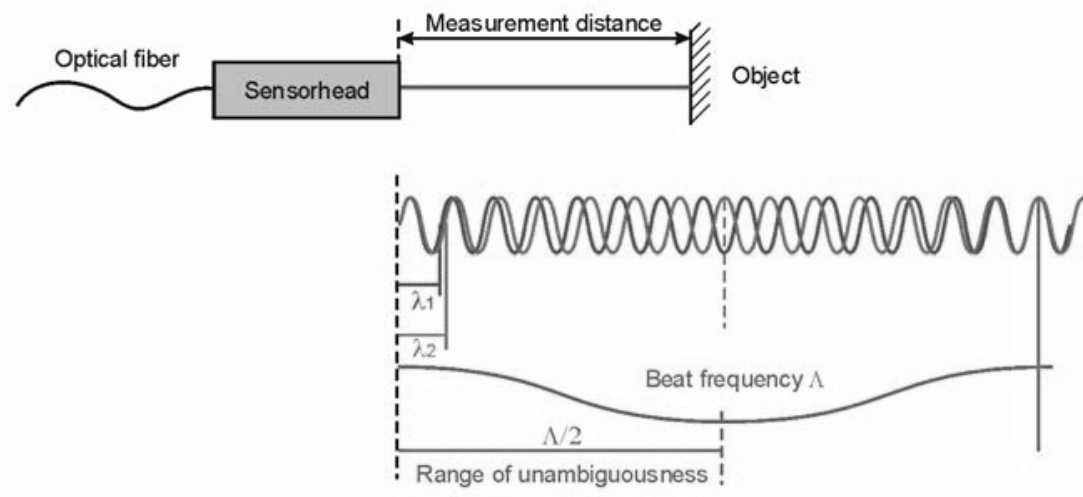

Fig. 1: Measurement principle of the MWLI.

The MWLI is based on an optical (contact-free) measurement scheme, applying the interferometric principle to measure distances even in comparably large working ranges with nanometer precision. While in other optical measurement systems (e.g. incoherent systems) the precision is inversly proportional to the working range (meaning either they comprise a high accuracy or a large working distance) due to the special interferometric scheme the precision of the MWLI principly is independent on the working distance. Furthermore the MWLI features a large dynamical (measurement) range of several centimeters and intervals of $2 \mathrm{~mm}$ in which an absolute determination of the position can be done. 
The fundamental measurement principle of the MWLI is the heterodyne interferometry. The distance to be determined is measured simultaneously by three independent interferometers, using closely adjacent wavelengths in the range around $\lambda=1550 \mathrm{~nm}$. The principle of superposition of optical waves allows to guide the signals in a conjoint optical fibre to the optical sensor head. From here the three signals leave the fibre and propagate to the object. Once reflected by the object the sensorhead incouples the three singals once more into the optical fibre where they are guided back to the analysis unit. Here, the individual optical phases of the three interferometric signals, created by the superimposition of the reflected signals and the according reference signal, are detected seperately and evaluated comparatively. With the information, that the different phase shifts of the three interferometer signals are caused by the same distance and the knowledge of the individual wavelengths of the beams the distance between the sensorhead and the object can be determined in a range of unambiguity much larger than the wavelength of each single signal. The basic physical principle exploited here is the analysis of the beating frequency of the used signals.

Following the measurement principle is explained using a sample of two wavelengths. The effective optical path difference results in a phase difference between signal and reference wave of each of the two waves, leading to a modulation of their interference pattern. Evaluating this intensity allows to conclude on the change of the optical path and so on the change in the distance to be measured. This however only can be done with an unambiguousness within the length of one half of a wavelength. When the difference of the two phase signals of different wavelengths is considered, the range of unambiguousness can be streched to the half of the value $\Lambda$, while $\Lambda$ can be calculated with:

$$
\Lambda=\frac{\lambda_{2} \cdot \lambda_{1}}{\lambda_{2}-\lambda_{1}}
$$

$\Lambda$ here also is named synthetic wavelength and is equal to the periode of the beating frequency of the two basic wavelengths $\lambda_{1}$ and $\lambda_{2}$. The smaller the distance between $\lambda_{1}$ and $\lambda_{2}$, the larger the resulting range of unambiguousness. Fig. 1 depicts the basic principle of the measurement. The distance is measured interferometrically by the two waves $\lambda_{1}$ and $\lambda_{2}$. Analyzing the individual phase of each of the measurements yields a position of the object with a precision of $\lambda / 2000$ within a range of half of the considered wavelengths $\left(\lambda_{1} / 2\right.$ or $\left.\lambda_{2} / 2\right)$. This allows a determination of the position of the object (or the length of the distance) with a precision in the nanometer range. Combining the individual phase values furthermore allows an explicit determination of the position within the range of half of the synthetic wavelength $\Lambda$. Therefore, high precision measurement within a large workingrange can be perfomed. Depending on the actual basic wavelengths this interval can be as large as $2 \mathrm{~mm}$ being more than thousand times larger than the range of unambiguousness of simple interferometers.

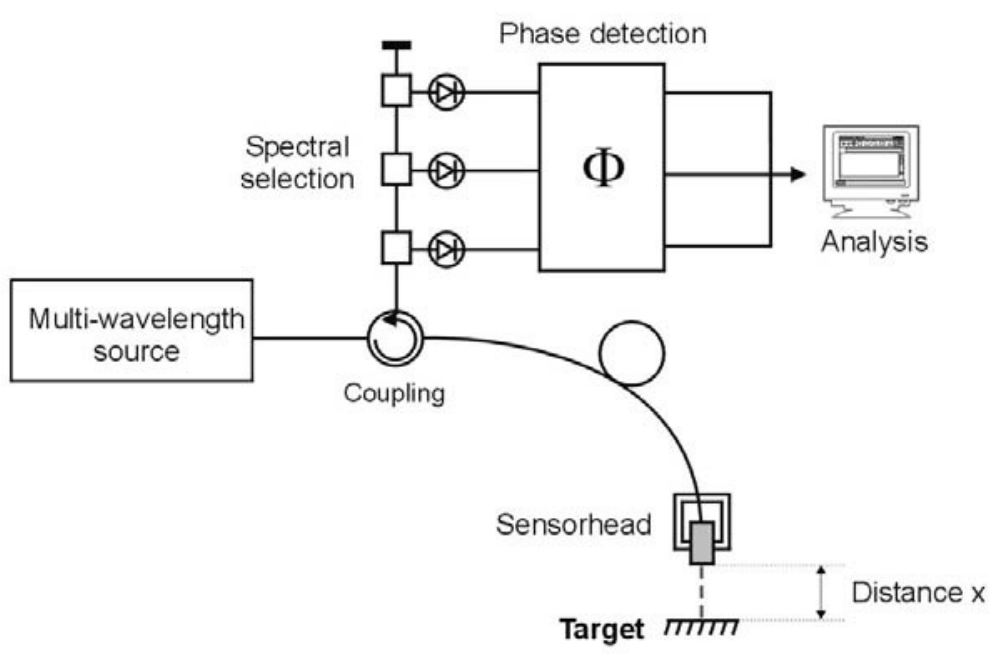

Fig. 2: Sketch up the setup of the MWLI-System. Due to the small fibre coupled sensorhead the measurement can be done far from the position of the analysation unit. 
A further explizit advantage of the enlarged range of unambiguousness is the ability to measure the topology of rough surfaces, which is impossible with simple interferometers. When light from high coherent light sources, as used in interferometers, is reflected by a rough surface with a roughness of Rz $\geq \lambda / 2$, the reflected signal is superimposed by a "coherent noise" (or speckle-pattern). This random pattern due to its $2 \pi$-ambiguity makes the evaluation of the correct phase and therefore the detection of the distance impossible. As the MWLI-system utilizes three independent interferometers in such a case also three individual speckle patterns for a certain object position resp. distance are created. The consideration of these patterns enables a correct determination of the distance and therefore even the optical measurement of the topology of rough surfaces in a large working range is possible.

The MWLI system is completely fibre coupled and with a sensorhead of only $18 \mathrm{~mm} \times 40 \mathrm{~mm}$ (diameter vs. length) a highly flexible integration of the sensor in different kind of industrial machinery and other applications is possible. Therefore, the measurement of the position of objects can be done where it is important. Errors and tolerances due to differences e.g. between high precision rulers and levers or mounts holding the object caused by mechanical stress or bending can be omitted. Including a calibration routine the quality control of the process can be performed within the machine itself. As these days high precision metrology measurement for quality control usually is done in external measurement systems, the repeatedly demounting and reassembling is a time consuming task implying several errors due to tolerances in position and angle. Adapting an high precision inline metrology measurement allows to eliminate the faults due to de- and reinstallation of the workpiece. Beyond that the fabrications process can be sped up several times.

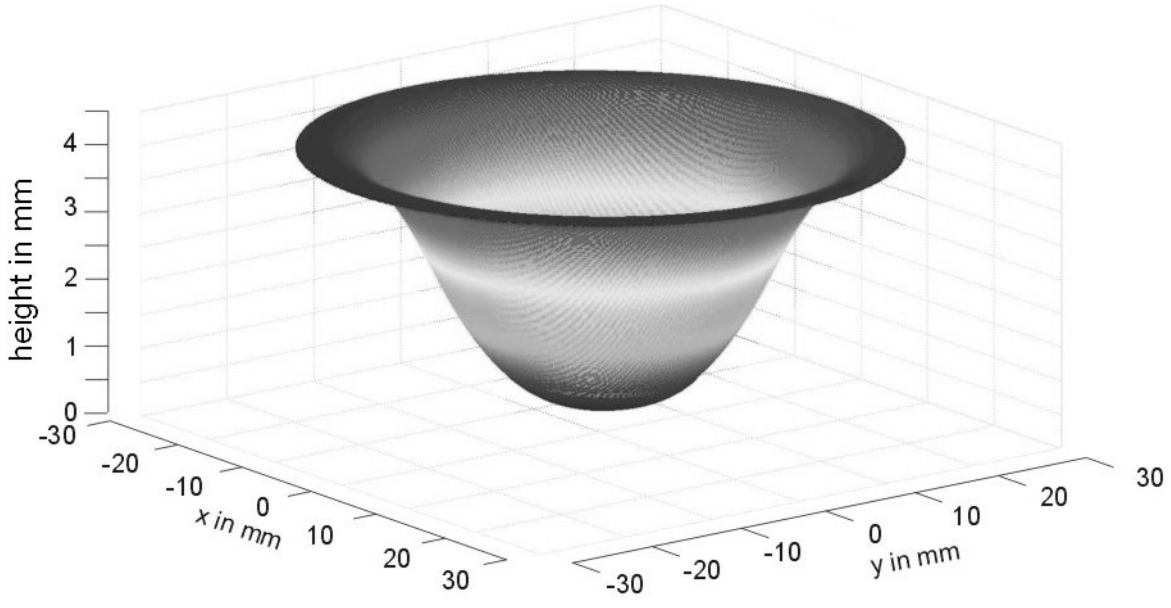

Fig. 3: Topology of a rough surface (grinded aspheric lens).

Fig. 3 shows the metrology curve of the surface of a grinded aspherical lens with a roughness of $\mathrm{Rz}=$ $3 \mu \mathrm{m}$. The scan was perfomed with a distance of $5 \mathrm{~mm}$ to $9 \mathrm{~mm}$ between the lens and the sensorhead while the sensorhead always was aligned perpendicular to the $x$-y-plane. Despite the rather large roughness and and the involved speckle pattern the topology of the object could be measured continuously within the dynamical range of the MWLI over a height distance of $4 \mathrm{~mm}$. While tactile systems can measure topologies only with direct contact to the surface, simple interferometers due to the roughness of the surface would fail in such metrology measurements.

In fig. 4 the topology measurement of a polished surface with a circular concave indentation with a diameter of $16 \mathrm{~mm}$ and a depth of $80 \mu \mathrm{m}$ is shown. In this measurement the distance between the sensorhead and the surface has been $5 \mathrm{~mm}$ and the sensor again was aligned perpendicular to the $x-y-$ plane. As can be seen in the figure the dip of $80 \mu \mathrm{m}$ is resolved, while the resolution of the measurement is well beyond $1 \mu \mathrm{m}$. 


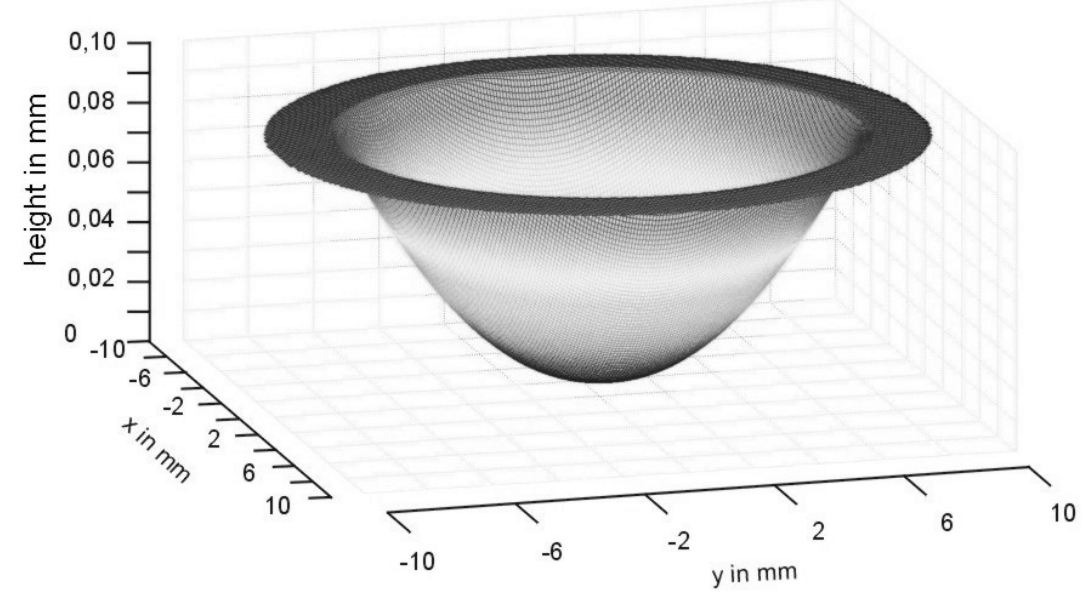

Fig. 4: Topology of a polished surface with an $80 \mu \mathrm{m}$ indentation

Fig. 5 impressively depicts the high precision of the MWLI-sensor system. Within a working distance of $25 \mathrm{~mm}$ the topology of a diamond grinded metallic mirrors was measured (Fig. 5a). The excerpt of the metrology curve in fig. $5 \mathrm{~b}$ shows variations in the surface of the mirror in the range of $20 \mathrm{~nm}$. These height variations stem from the tracks of the turning diamond during the manufacturing process of the mirror. Deviations of such extend are hardly detectably by tacticle measurement systems.
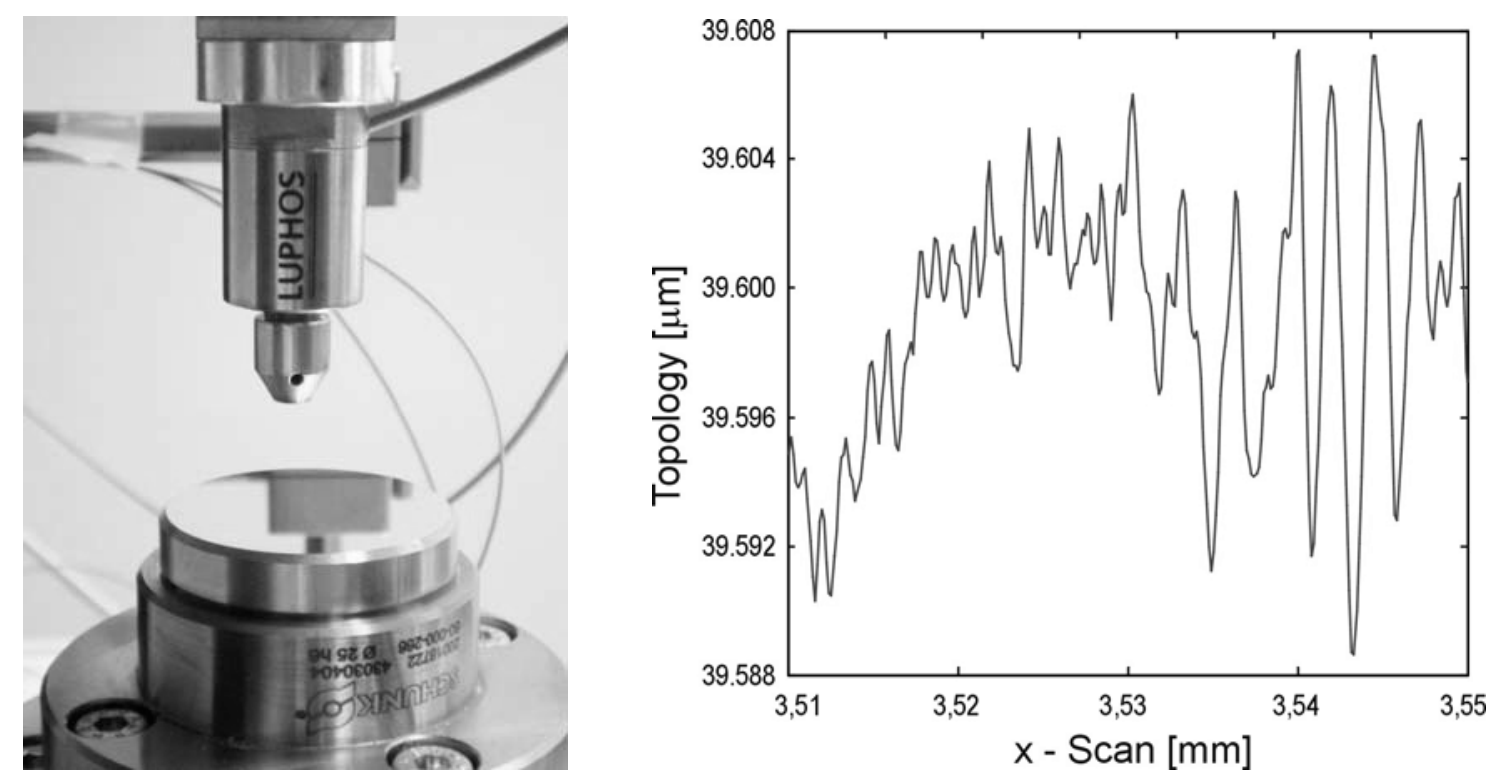

Fig. 5a: The sensorhead of the MWLI measuring a diamond grinded mirror

Fig. 5b: Excerpt of the measurement depicted in 5a.

The MWLI resolves scratches with a resolution of $1 \mathrm{~nm}$.

The high precision in combination with the high flexibility makes the MVLI-sensor system a versatile tool for a large variety of measurement tasks in industrial fabrication as well as in research. Being able to provide a measurment in large working distances with an accuracy in the nanometer range the MWLI offers features, that were not available so far. 